\title{
Cistos primários do epitélio pigmentar da íris e corpo ciliar: aspectos de biomicroscopia ultra-sônica
}

\author{
Primary cysts of the iris and ciliary body pigment epithelium: ultrasound biomicroscopy \\ features
}

Bernadete Ayres ${ }^{(1)}$

Norma Allemann ${ }^{(2)}$

Celia Nakanami ${ }^{(3)}$

Consuelo B. D. Adan (4)

\section{RESUMO}

Objetivo: Descrever as características, incidência e distribuição dos cistos primários de epitélio pigmentar de íris e corpo ciliar ao exame de biomicroscopia ultra-sônica, que devem ser diferenciados de lesões sólidas.

Métodos: Foram estudados de modo retrospectivo os prontuários de 73 pacientes, 82 olhos, com diagnóstico ecográfico de cisto primário de íris ou corpo ciliar durante o período de janeiro/97 a dezembro/99. Utilizou-se o biomicroscópio ultra-sônico, aplicando técnicas padronizadas de imersão.

Resultados: À biomicroscopia ultra-sônica os cistos caracterizaramse por apresentarem paredes finas e regulares, e conteúdo anecóico. Quarenta e oito pacientes $(65,7 \%)$ eram do sexo feminino. A maior incidência $(28,8 \%)$ ocorreu para o grupo incluído no intervalo de 20 a 29 anos de idade. Observou-se uma característica distribuição, predominantemente nos quadrantes temporais inferiores.

Conclusões: A biomicroscopia ultra-sônica mostrou-se útil no diagnóstico de cistos primários do epitélio pigmentar da íris e do corpo ciliar, auxiliando na diferenciação de patologias tumorais e avaliando possíveis complicações. $O$ conhecimento dos critérios ecográficos e da distribuição epidemiológica facilitam o diagnóstico destas lesões.

Palavras-chave: Ultra-som; Cistos; Íris e corpo ciliar; Epitélio pigmentar.

\section{INTRODUÇÃOO}

Os cistos de íris e de corpo ciliar podem ser classificados em primários ou secundários. Cistos primários, em geral, originam-se do epitélio pigmentar e, muito raramente, a partir do estroma ${ }^{1}$.

Cistos primários do epitélio pigmentar da íris e do corpo ciliar são de ocorrência rara.

Histologicamente, estruturam-se como cavidade cística preenchida por fluido seroso, delimitada por uma parede constituída de células epiteliais ${ }^{2}$. Geralmente são lesões múltiplas, encontradas predominantemente nos quadrantes inferiores e temporais, com dimensões estáveis e que não requerem tratamento. O diagnóstico diferencial é feito com lesões tumorais do corpo ciliar e íris ${ }^{3}$ e com lesões císticas como o cisto primário do estroma iriano e o cisto de implantação secundária de câmara anterior (associados a traumatismo ou cirurgia prévias). Clinicamente estes cistos são observados ao exame com lâmpada de fenda e gonioscopia, porém, em alguns casos, 
apresentam-se inacessíveis às técnicas convencionais. A biomicroscopia ultra-sônica (UBM) é um método de imagem, não invasivo, que usa um transdutor de alta frequência (50 $\mathrm{MHz})$ e alta resolução $(20 \mu \mathrm{m})$, porém com pouca penetração (aproximadamente $5 \mathrm{~mm}$ ), sendo eficaz para a avaliação do segmento anterior do globo ocular ${ }^{4,5}$.

\section{MATERIAL E MÉTODOS}

Foram estudados de modo retrospectivo os prontuários de 73 pacientes, 82 olhos, com diagnóstico ecográfico de cisto primário de íris ou corpo ciliar no Setor de Ultra-sonografia do Departamento de Oftalmologia, Universidade Federal de São Paulo - Escola Paulista de Medicina, dentro de um número total de 2160 exames realizados durante o período de janeiro/97 a dezembro/99. Foram excluídos da amostra os casos de trauma ocular, cirurgia ocular prévia e os cistos associados a nevos ou melanoma. Foram analisadas as seguintes variáveis relativas aos pacientes: sexo, idade, olho afetado, profundidade da câmara anterior e aspecto do ângulo camerular. Em relação aos cistos, foram pesquisados: a localização, a dimensão e o número de lesões. Para a localização, o critério usado foi a distribuição por meridianos horários.

Foi utilizado o biomicroscópio ultra-sônico (Modelo 840, Humphrey Instruments, Califórnia, USA) com transdutor de aproximadamente $50 \mathrm{MHz}$, que proporciona uma resolução aproximada de $20 \mu \mathrm{m}$ e penetração tecidual de $5 \mathrm{~mm}$. Os pacientes foram examinados em decúbito dorsal horizontal. Após instilação de anestésico tópico (cloridrato de tetracaína com fenilefrina, Allergan-Frumtost, São Paulo), uma cuba ocular de imersão foi colocada entre as pálpebras e preenchida com substância viscoelástica (metilcelulose a $2 \%$, Ophthalmos, São Paulo). A sonda era colocada em contato com a substância viscoelástica e direcionada perpendicularmente em posição axial à câmara anterior, assim como em posições radial e transversal para avaliação das secções em relação às estruturas angulares, denominadas de acordo com o meridiano horário. As imagens foram arquivadas e, posteriormente, mensurações e descrições puderam ser obtidas.

\section{RESULTADOS}

Na presente casuística foram examinados 82 olhos de 73 pacientes. Desses pacientes, $48(65,7 \%)$ eram do sexo feminino e $25(34,3 \%)$ do sexo masculino. Por simplicidade, cada olho foi considerado de forma independente, mesmo considerando que em 9 pacientes ocorreu bilateralidade dos achados. Foi observado predomínio do sexo feminino $\left(\chi^{2}, \mathrm{p}=\right.$ 0,0204, correção de Yates) na amostra.

A idade dos pacientes analisados variou entre 0,33 e 87 anos, com média de 39,1 $\pm 20,1$ anos. As freqüências observadas foram distribuídas por faixa etária, conforme demonstrado na figura 1 . A maior incidência $(28,8 \%)$ ocorreu para o grupo incluído no intervalo de 20 a 29 anos de idade (figura 1). Um teste de aderência mostrou a existência de diferenças significativas nas incidências por faixa etária $\left(\chi^{2}, \mathrm{p}=0,0046\right)$. Não houve diferença significante de faixa etária entre os pacientes do sexo feminino e masculino (teste " $t$ ", $p=0,2818$ ).

Ao exame de UBM, os cistos primários de epitélio pigmentar de íris e corpo ciliar caracterizaram-se por apresentarem uma parede fina, regular, lisa, de média refletividade com conteúdo anecóico (figura 2).

Não houve diferença significante ( $p=0,3203$, correção de Yates) entre as ocorrências de cistos nos olhos direitos e esquerdos.

Para a medida da profundidade da câmara anterior foram excluídos os casos de pacientes na faixa etária de 0 a 9 anos. Foi obtido nessa amostragem um valor médio de profundidade de câmara anterior de 2,81 $\pm 0,48 \mathrm{~mm}$ (variação de 1,56 mm a $3,83 \mathrm{~mm}$ ).

$\mathrm{O}$ estudo do ângulo camerular revelou 40 pacientes com ângulo aberto e 40 pacientes com estreitamento e/ou fechamento focal (figura $2 \mathrm{~d}$ ), ou seja, correspondente à topografia do cisto. Não ocorreu diferença significante entre as incidências ( $\mathrm{p}>0,05$, correção de Yates). Foram observados 2 casos $(2,4 \%)$ de fechamento de ângulo por $360^{\circ}$, não relacionado à topografia dos cistos.

Foram detectados $48(60 \%)$ casos de cistos múltiplos (figura 3$)$ e $32(40 \%)$ de cistos únicos. Não houve diferença significante entre as freqüências para os dois tipos de ocorrência $\left(\chi^{2}, \mathrm{p}=0,094\right.$, correção de Yates).

A dimensão do cisto foi avaliada tomando-se sua altura, que variou entre 200 a $2500 \mu \mathrm{m}$, com valor médio de $917 \pm$ $448 \mu \mathrm{m}$. Essas alturas foram correlacionadas com a idade dos pacientes. Concluiu-se que a altura do cisto independe da idade do paciente $\left(\mathrm{R}^{2}=0,0034, \mathrm{p}=0,2780\right)$.

Quanto à localização do cistos, foi analisado o número de

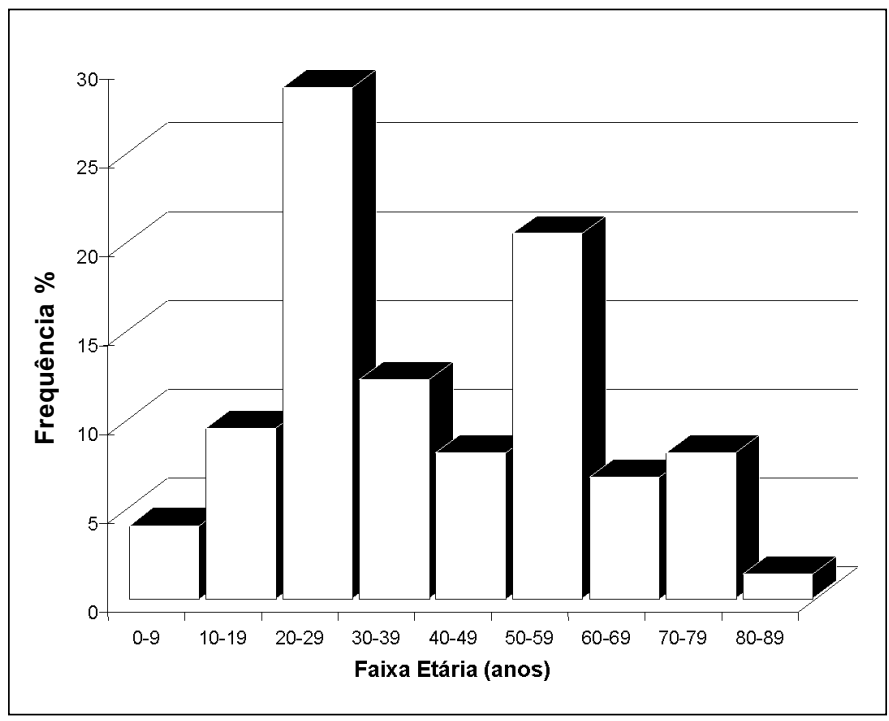

Fig. 1 - Histograma demonstrativo da incidência de cistos primários de íris e corpo ciliar por faixa etária. 


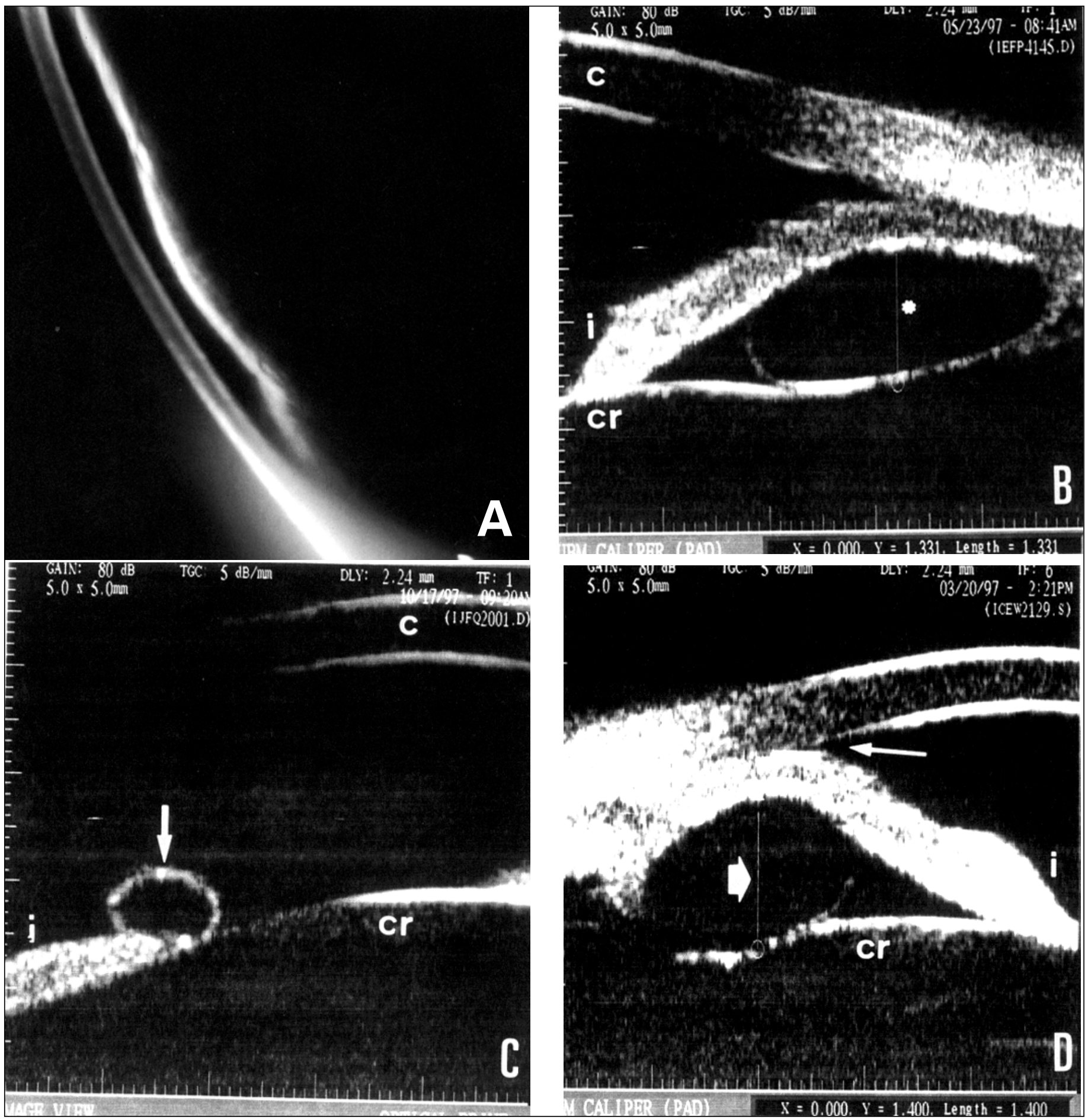

Fig. 2 - A. fotografia clínica em lâmpada de fenda mostrando o abaulamento anterior da íris periférica provocado por cisto primário retro-iriano. B. imagem de biomiroscopia ultra-sônica mostrando cisto primário (junção irido-ciliar) com parede fina e conteúdo anecóico (asterisco); C. pequeno cisto em borda pupilar (seta); D. grande cisto, altura=1,4 mm (length, seta menor) na junção irido-ciliar provocando fechamento angular (seta maior). C=córnea, CR=cristalino, l=íris.

meridianos afetados em cada caso. Observou-se uma característica distribuição, predominantemente nos quadrantes temporais inferiores, com 70 ocorrências $(57,4 \%)$ nos meridianos de $6,7,8$ e 9 em olho direito $\left(\chi^{2}, p=0,0001\right)$ e 53 ocorrências
$(51,5 \%)$ em $3,4,5$ e 6 em olho esquerdo $\left(\chi^{2}, p=0,0499\right)$ (figura 4).

$\mathrm{Na}$ série deste estudo pôde-se observar um paciente referido com diagnóstico de glaucoma e síndrome de íris em 

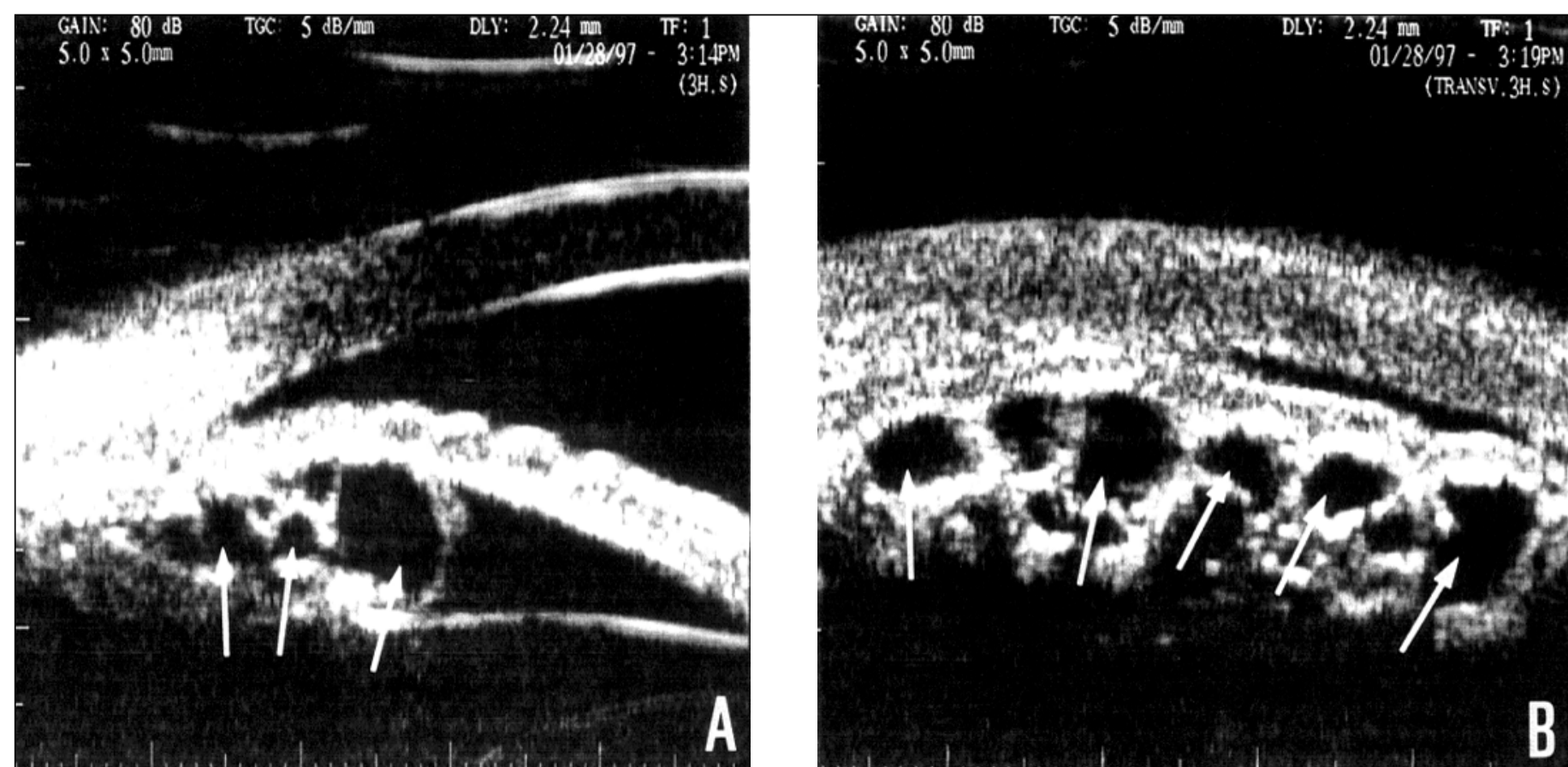

Fig. 3 - Imagem de biomicroscopia ultra-sônica apresentando múltiplos cistos primários de íris (setas). A. incidência longitudinal; B. incidência transversal.

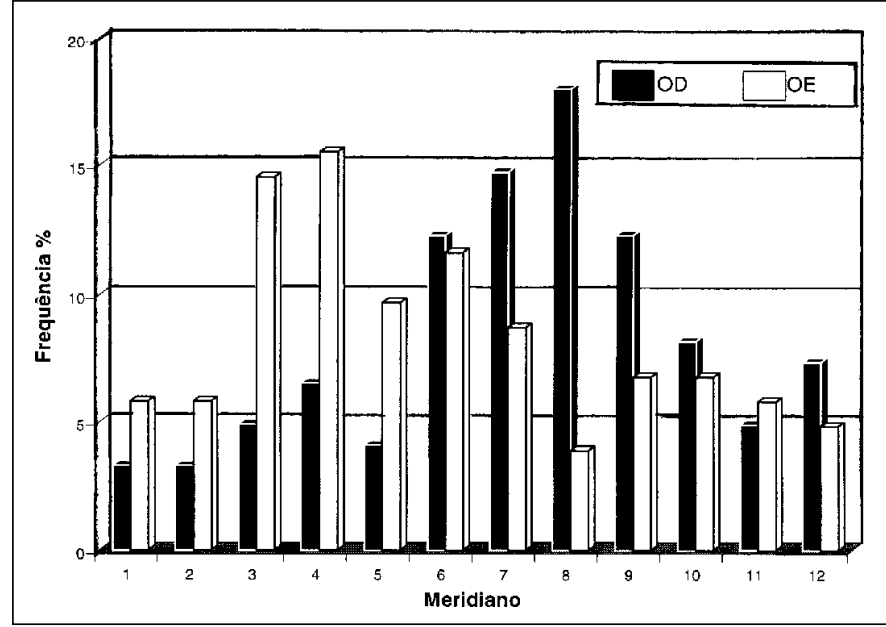

Fig. 4 - Histograma demonstrativo da incidência de cistos primários de íris e corpo ciliar e sua localização por meridianos.

plateau. O UBM revelou bilateralmente câmara anterior rasa, com fechamento angular por $360^{\circ}$ e múltiplos cistos irianos em olho direito, localizados nos meridianos de 8,9 e 10 , e, em olho esquerdo, nos meridianos de 3, 4, 5 e 6; a íris apresentava-se retificada e o corpo ciliar anteriorizado. Notou-se também iridectomia a laser em ambos olhos, procedimento realizado previamente para controle da pressão intra-ocular. Em nosso estudo pôde-se observar as imagens ecográficas de um paciente portador de múltiplos cistos em íris e corpo ciliar, acometendo os meridianos de 3, 4, 7, e 8, sendo que este último apresentava fechamento angular. $\mathrm{O}$ paciente foi sub- metido à ruptura do cisto de maior dimensão (altura=1,4 mm) com Nd:YAG laser e conseqüente colapso da cavidade cística e enrrugamento da parede (altura após aplicação $=0,394 \mathrm{~mm}$ ), com abertura do ângulo camerular (figura 5).

Dentre os pacientes examinados, um apresentou formações císticas, múltiplas, bilaterais, e o cisto de maior volume apresentava parede irregular, supostamente colapsada, com suspeita de ruptura espontânea do cisto, já que não havia história pregressa de intervenção cirúrgica (figura $5 \mathrm{c}$ ).

Outro caso interessante observado foi um cisto primário de íris localizado em borda pupilar, com configuração pedunculada, cuja extremidade livre assumia diferentes posições de acordo com o movimento ocular (figura 6).

\section{DISCUSSÃO}

Os cistos primários do epitélio pigmentar da íris e do corpo ciliar são achados ocasionais. Shields ${ }^{1}$ propôs uma classificação para os cistos irianos, baseada em sua localização na íris: central (pupilar), de zona média, periféricos (junção iridociliar) e deslocados (livre).

A exata patogênese destes cistos não é bem definida, embora existam duas teorias propondo sua origem. Vail e Merz ${ }^{6}$ sugeriram que tais cistos representam a separação entre as duas camadas epiteliais resultante da tração exercida pelos ligamentos zonulares. Outra teoria propõe que os cistos são formados pela proliferação de tecido epitelial ${ }^{7}$.

A ocorrência da patologia parece ser esporádica, entretanto, a ocorrência familial de cistos múltiplos associados a 


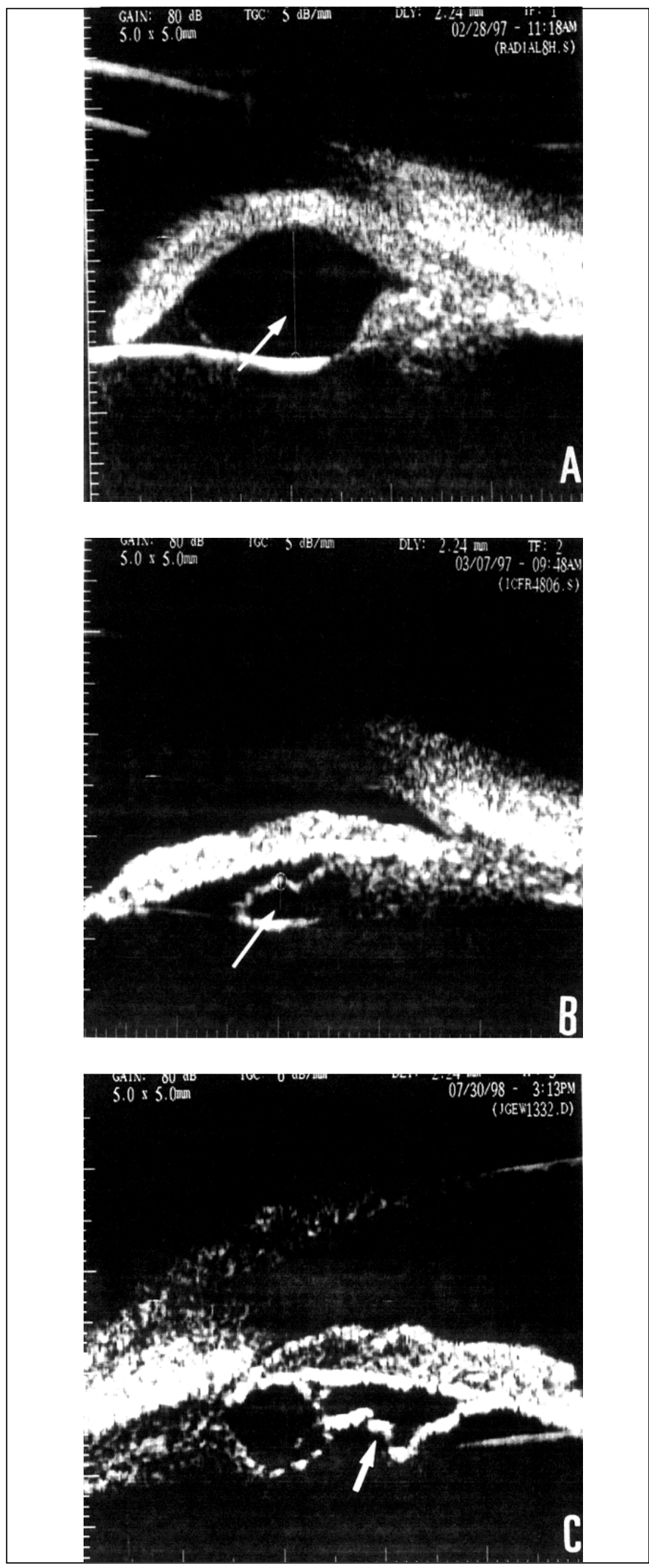

Fig. 5 - Imagem de biomicroscopia ultra-sônica de paciente submetido a ruptura de cisto com $\mathrm{Nd}$ :YAG laser: $A$. pré-aplicação (altura $=1,4 \mathrm{~mm}$, vide seta) e B. após aplicação (altura $=0,3 \mathrm{~mm}$, vide seta), notar colapso do cisto e abertura do ângulo camerular; C. caso suspeito de ruptura espontânea devido a irregularidade da parede (seta). glaucoma, sugerindo herança autossômica dominante já foi publicada ${ }^{8}$. Em nossa série os familiares dos afetados não foram investigados, portanto a possibilidade do acometimento familial não pode ser excluída.

Ao exame de UBM, os cistos primários de epitélio pigmentar de íris e corpo ciliar caracterizam-se por apresentarem uma parede fina, regular, lisa, de média refletividade e conteúdo anecóico. Prévios estudos demonstraram que o número de cistos primários periféricos de íris detectados ao exame com UBM é maior do que o esperado aos exames clínicos convencionais ${ }^{9}$.

Em nosso estudo foi observado o predomínio em pacientes do sexo feminino e a localização predominante nos quadrantes temporais, concordando com a mesmas observações de Shields ${ }^{10}$ em uma série de 62 pacientes portadores de cistos primários irianos.

Lois ${ }^{3}$ reportou uma maior incidência em adultos jovens, com idade média 33 anos. Em nossa pesquisa a maior incidência $(28,8 \%)$ ocorreu para o grupo incluído no intervalo de 20 a 29 anos de idade, concordando com o relato anterior.

Em nossa série não encontramos diferença significativa entre a ocorrência de cistos únicos e múltiplos. É provável que a maior incidência de cistos múltiplos $(60 \%)$ pudesse ficar comprovada com uma amostra de maior tamanho.

Os cistos primários de epitélio pigmentar podem apresentar dimensões diversas. Nesta casuística encontrou-se altura dos cistos variando entre 200 a $2500 \mu \mathrm{m}$. Interessante observar que quando estes cistos alcançam grandes dimensões provocam abaulamento anterior da raiz da íris provocando um estreitamento ou até mesmo fechamento angular. Ainda em relação ao tamanho, concluiu-se que a altura do cisto independe da idade do paciente. Kunimatsu ${ }^{11}$, em estudo prospectivo reportou diminuição do tamanho médio dos cistos de corpo ciliar com o decorrer da idade.

A evolução natural dessas formações raramente pode levar a complicações, tais como: toque corneal, subluxação do cristalino, catarata focal, irite, e glaucoma. Neste levantamento observamos um paciente referido com diagnóstico de glaucoma com síndrome de íris em plateau e que apresentou achados bilaterais peculiares ao exame com UBM (câmara anterior rasa, retificação do plano iriano e corpo ciliar anteriorizado), além de múltiplos cistos de íris. Em recente publicação encontrou-se 3 casos de síndrome de íris em plateau associados com múltiplos cistos e diagnosticados com UBM, sugerindo que tais cistos poderiam ser responsáveis, pelo menos parcialmente, pela patogênese do quadro, tornando o ângulo passível de fechamento ${ }^{12}$.

Estes cistos raramente requerem tratamento. Em casos de procedimentos como o uso de Nd:YAG laser, as imagens obtidas pela UBM no pré-operatório e após a sua aplicação revelaram a eficácia do tratamento, mostrando o colapso do cisto após sua ruptura.

O diagnóstico diferencial dos cistos primários de íris e corpo ciliar inclui: tumores, cisto do estroma iriano e cisto de implantação secundária de íris de câmara anterior. Os tumores 


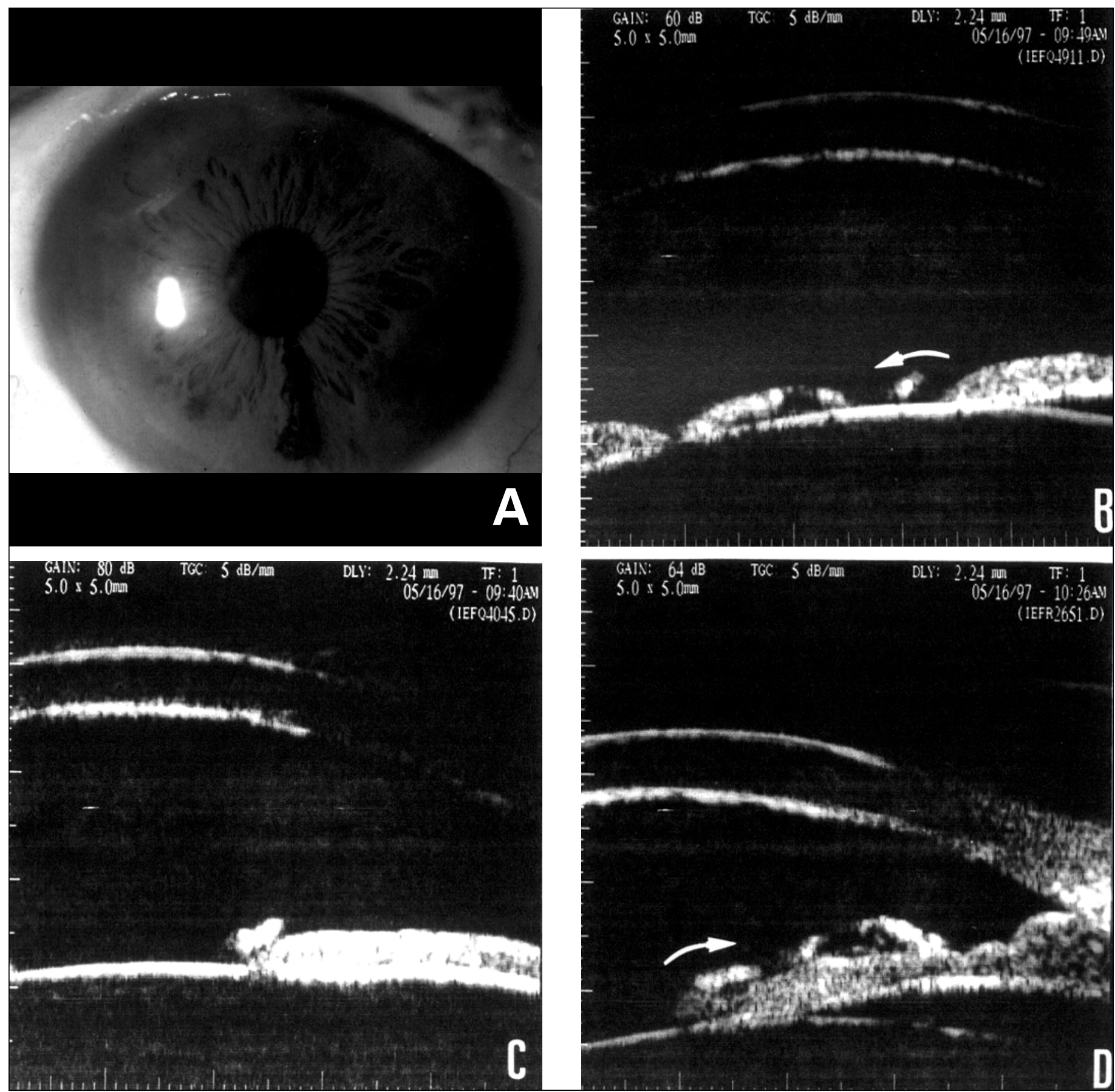

Fig. 6 - A. fotografia em lâmpada de fenda de lesão cística de borda pupilar às 6 horas, que se projeta para a câmara anterior e apresenta superfície irregularmente pigmentada; Imagem de biomicroscopia ultra-sônica do caso com diferentes angulações do globo ocular (setas demonstrativas da mobilidade): B. supraversão, C. posição primária do olhar e D. infraversão. Observar a parede fina, porém irregular (despigmentação da parede do cisto) e o conteúdo anecóico.

apresentam-se ao UBM como lesões sólidas ${ }^{13}$. Cistos do estroma iriano projetam-se para a câmara anterior e apresentam parede espessa ${ }^{14}$. Os cistos de implantação secundária de câmara anterior, à UBM, caracterizam-se como lesões unilaterais, únicas, de conteúdo hipodenso (ou com partículas em suspensão) ou dispostas em camadas hiper-refletivas, com delaminação do estroma iriano ou ainda livres na câmara anterior ${ }^{15}$.
Em resumo, os cistos primários do epitélio pigmentar da íris e corpo ciliar ocorrem predominantemente em pacientes adultos jovens, preferencialmente do sexo feminino e localizam-se principalmente nos quadrantes temporais inferiores. Possuem características peculiares à biomicroscopia que permitem sua identificação e diferenciação de lesões sólidas. A biomicroscopia ultra-sônica revelou-se um método útil e não 
invasivo na detecção e mensuração destas formações e na avaliação de sua relação com outras estruturas oculares do segmento anterior do globo ocular.

\section{SUMMARY}

Purpose: To describe the ultrasound biomicroscopic characteristics, incidence, distribution and location of primary cysts of the iris and ciliary body pigment epithelium, and to differentiate them from solid lesions.

Methods: A retrospective study was performed through a review of charts of 73 patients, 82 eyes, with echographic diagnosis of primary cysts of the iris and ciliary body pigment epithelium during a 36-month period (January/97 through December/99). All examinations were performed using an ultrasound biomicroscope applying standard immersion techniques.

Results: Ultrasound biomicroscopy revealed typical findings of the primary cysts of the pigment epithelium such as thin and regular walls with no internal content. Incidence was higher in individuals included in the interval of 20 to 29 years of age (28.8\%). Forty-eight patients (65.7\%) were female. The cysts were found most frequently in the inferior and temporal sectors.

Conclusions: Ultrasound biomicroscopy was a useful tool for evaluating cystic lesions of the iris and ciliary body, also valuable in differentiating these lesions from anterior segment tumors and evaluating potential complications. The knowledge of the echographic criteria and epidemio- logical characteristic can assist in the diagnosis of these lesions.

Keywords: Ultrasound; Cysts; Iris and ciliary body; Pigment epithelium.

\section{REFERÊNCIAS BIBLIOGRÁFICAS}

1. Shields JA. Primary cysts of the iris. Trans Am Ophthalmol Soc 1981;79:771-809

2. Hogan MJ, Zimmerman LE. Ophthalmic pathology: cyst of the iris and ciliary body. Philadelphia: W.B. Saunders, 1962;367-72.

3. Lois N, Shields CL, Shields JA, Mercado G. Primary cysts of the iris pigment epithelium. Ophthalmology 1998;105:1879-85.

4. Pavlin CJ, Harasiewicz K, Sherar MD, Foster FS. Clinical use of ultrasound biomicroscopy. Ophthalmology 1991;98:287-95.

5. Allemann N. Biomicroscopia ultra-sônica. Arq Bras Oftalmol 1995;58:283-5.

6. Vail D, Merz EH. Embryonic intraepithelial cyst of the ciliary body processes. Am J Ophthalmol 1951;35:1676-84.

7. Kozart DM, Sheie HG. Spontaneous cysts of the ciliary epithelium. Ophthalmology 1970;74:534-47.

8. Vela A, Rieser JC, Campbell DG. The heredity and treatment of angle-closure glaucoma secondary to iris and ciliary body cysts. Ophthalmology 1983; 91:332-7.

9. Augsburger JJ, Affel LL, Benarosh DA. Ultrasound biomicroscopy of cystic lesions of the iris and ciliary body. Trans Am Ophthalmol Soc 1996;94:259-74.

10. Shields JA, Kline MW, Augsburger JJ. Primary iris cysts: A review of the literature and report of 62 cases. Br J Ophthalmol 68:152,1984.

11. Kunimatsu S, Araie M, Ohara K, Hamada C. Ultrasound biomicroscopy of ciliary body cysts. Am J Ohphthamol 1999;127:48-55.

12. Azuara-Blanco A, Spaeth GL, Araujo SV, Augsburger JJ, Terebuh AK Plateau iris syndrome associated with multiple ciliary body cysts. Arch Opthalmol 1996;114:666-8.

13. Pavlin CJ, McWhae JA, McGowan HD, Foster FS. Ultrasound biomicroscopy of anterior segment tumors. Ophthalmology 1992;99:1220-8.

14. Lois N, Shields CL, Shields JA, Mercado, De Potter P. Primary iris stromal cysts. Ophthalmology 1998;105:1317-22.

15. Adan CD, Chojniak MM, Allemann N. Ultrasound biomicroscopy and secondary epithelial downgrowth cysts of anterior chamber. Arq Bras Oftalmol 1998;61:656-61.

\section{Congresso da Sociedade Brasileira de Retina e Vítreo}

\section{Imperial Othon Hotel - Fortaleza - CE}

\section{7 a 29 de Abril de 2.001}

\section{INFORMAC̣ÕES: ARX Promoções e Eventos}

Telf.: (0xx85) 272-1572 - Fax: (0xx85) 272-7795 